\title{
Id Gene Regulation and Function in the Prosensory Domains of the Chicken Inner Ear: A Link between Bmp Signaling and Atoh1
}

\author{
Andrés Kamaid, ${ }^{\star}$ Joana Neves, ${ }^{\star}$ and Fernando Giráldez \\ Department de Ciències Experimentals i de la Salut, Universitat Pompeu Fabra, Parc de Recerca Biomèdica de Barcelona, 08003-Barcelona, Spain
}

\begin{abstract}
Bone morphogenetic proteins (Bmps) regulate the expression of the proneural gene Atoh1 and the generation of hair cells in the developing inner ear. The present work explored the role of Inhibitor of Differentiation genes (Id1-3) in this process. The results show that Id genes are expressed in the prosensory domains of the otic vesicle, along with $B m p 4$ and $B m p$. Those domains exhibit high levels of the phosphorylated form of Bmp-responding R-Smads (P-Smad1,5,8), and of Bmp-dependent Smad transcriptional activity as shown by the BRE-tk-EGFP reporter. Increased Bmp signaling induces the expression of Id1-3 along with the inhibition of Atoh1. Conversely, the Bmp antagonist Noggin or the Bmp-receptor inhibitor Dorsomorphin elicit opposite effects, indicating that Bmp signaling is necessary for Id expression and Atohl regulation in the otocyst. The forced expression of Id3 is sufficient to reduce Atoh1 expression and to prevent the expression of hair cell differentiation markers. Together, these results suggest that $I d s$ are part of the machinery that mediates the regulation of hair cell differentiation exerted by Bmps. In agreement with that, during hair cell differentiation Bmp4 expression, P-Smad1,5,8 levels and $I d$ expression are downregulated from hair cells. However, $I d s$ are also downregulated from the supporting cells which contrarily to hair cells exhibit high levels of $B m p 4$ expression, P-Smad1,5,8, and BRE-tk-EGFP activity, suggesting that in these cells Ids escape from Bmp/Smad signaling. The differential regulation of Ids in time and space may underlie the multiple functions of Bmp signaling during sensory organ development.
\end{abstract}

\section{Introduction}

Hair cells of the inner ear are responsible for the initial step in the neural processing of sound and balance in vertebrates. They originate from the prosensory domains of the otic vesicle and their commitment to a specific lineage follows a stereotyped spatial and temporal sequence (Fritzsch et al., 2006; Abello and Alsina, 2007; Bell et al., 2008). The proneural gene Atohl codes for a basic helix-loop-helix (bHLH) transcription factor that behaves as a master gene for hair cell specification, Atohl being necessary and sufficient for hair cell generation (Bermingham et al., 1999; Zheng and Gao, 2000). However, the mechanisms that regulate the expression of Atoh 1 and the onset of hair cell differentiation in sensory patches are largely unknown.

\footnotetext{
Received May 20, 2010; accepted July 1, 2010.

This work was supported by Ministerio de Ciencia e Innovación, Spain Grants BFU-2008-00714 and PLE-20090098, and a fellowship from Fundação para a Ciência ea Tecnologia, SFRH/BD/25688/2005, to J.N. We thank Cristina Pujades, Thomas Schimmang, Malcolm Maden, Domingos Henrique, and Lena Gunhaga for reading the manuscript and comments, and Marta Linares for excellent technical assistance. The help of Mireia Chamizo is also greatly acknowledged. Thomas Schultheiss and Elisa Martí (Institut de Biologia Molecular de Barcelona-Consejo Superior de Investigaciones Científicas, Barcelona, Spain) kindly shared their expression constructs with us, and Edward Laufer (Columbia University, New York, NY) the P-Smad1,5,8 antibody. MyoVIlla monoclonal antibody was obtained from the Developmental Studies Hybridoma Bank under the auspices of the National Institute of Child Health and Human Development and maintained by the University of lowa, Department of Biological Sciences (lowa City, IA).

${ }^{*}$ A.K. and J.N. contributed equally to the work.

Correspondence should be addressed to Fernando Giráldez, CEXS-Universitat Pompeu Fabra, PRBB, c/Dr. Aiguader 88, 08003-Barcelona, Spain. E-mail: fernando.giraldez@upf.edu.

A. Kamaid's present address: Instituto de Fisiologia Celular, Universidad Nacional Autónoma de México, Ciudad Universitaria, Circuito Exterior S/N, México City, 04510 D.F., Mexico.

D01:10.1523/JNEUROSCI.2570-10.2010

Copyright $\odot 2010$ the authors $\quad$ 0270-6474/10/3011426-09\$15.00/0
}

Bone morphogenetic proteins (Bmps) regulate essential processes in neural development (Hogan, 1996; Massagué et al., 2005). Several Bmp ligands are expressed in the developing ear where they map to prosensory patches and sensory organs in several animal species (Oh et al., 1996; Morsli et al., 1998). The functions of Bmp signaling in the inner ear are probably multiple and span across different developmental stages (Chang et al., 1999, 2002, 2008; Gerlach et al., 2000; Li et al., 2005; Pujades et al., 2006). With respect to sensory organ development, Bmps are known to prevent Atoh1 expression and to maintain the undifferentiated state of sensory progenitors (Pujades et al., 2006). This function is reminiscent of that of Bmps in other neural progenitors and stem cells where they maintain the pluripotency of embryonic stem cells and have negative effects on neural differentiation (Varga and Wrana, 2005; Chen and Panchision, 2007). In addition, Bmp signaling is also necessary for the specification of nonsensory and supporting cell fates from the prosensory domain (Chang et al., 2008). These diverse and sometimes paradoxical functions may be related to the specific regulation of the Bmp response in different cellular states and contexts.

Bmps regulate the expression of Inhibitor of Differentiation and DNA binding $(I d)$ in various cell types (Ruzinova and Benezra, 2003). Id proteins are dominant-negative regulators of bHLH factors that promote self renewal and inhibit differentiation (Benezra et al., 1990; Norton, 2000). Recently, Id3 has been shown to counteract hair cell differentiation in mouse cochlear explants (Jones et al., 2006), suggesting that Ids may regulate Atoh1 expression in the ear sensory epithelium. However, little is known about $I d$ expression, regulation and function in the 
prosensory epithelium, and whether $I d s$ are related to the initiation of hair cell differentiation.

In the present work we have explored the relationship between Id genes and the prosensory function of Bmps, and how this relates to the onset of Atoh 1 expression and the generation of hair cells.

\section{Materials and Methods}

Embryos. Fertilized hens' eggs (Granja Gibert) were incubated at $38^{\circ} \mathrm{C}$ for designated times and embryos were staged according to the system of Hamburger and Hamilton (1992).

In situ hybridization. For in situ hybridization (ISH), embryos were dissected in PBS, fixed overnight in $4 \%$ paraformaldehyde, and processed according to (Wilkinson and Nieto, 1993). For stages embryonic day 4 (E4)-E7, ISH was performed on cryostat sections. The protocol was performed using the automated system from Insitu Pro VS (Intavis AG, Bioanalytical Systems). Double ISH hybridization was performed by labeling of Id3 probe with digoxigenin and Atoh1 probe with fluorescein (RNA labeling kit, Roche). The protocol and conditions for hybridization were identical to the single ISH, except for development of color. Fluo-Atoh1 was developed first, using the tyramide-CY3 fluorescent system (TSA Cyanine 3 System, PerkinElmer). Slides were analyzed under fluorescent microscope, and then processed for NBT/BCIP for color development of the Id3 probe. Riboprobes were as follows: Atoh1 (BSRC chick EST) Bmp4 and Bmp7 (Elisa Piedra et al., 2000), and Id1-3 (Kee and Bronner-Fraser, 2001a,b,c).

Electroporation. Focal electroporation of HH20-HH21 otic vesicles was performed in ovo, using a method modified from Chang et al. (2008). The cathode consisted of a $0.3 \mathrm{~mm}$ diameter Pt tip attached to a handle and the anode was a $0.5 \mathrm{~mm}$ diameter Pt electrode that was placed underneath the embryo. DNA was injected into the otic vesicle at a concentration of $6-8 \mu \mathrm{g} / \mathrm{ml}$ and electroporation conditions were 8 pulses of $10-12 \mathrm{~V}, 250 \mathrm{~ms}, 50 \mathrm{~Hz}$. Embryos were allowed to develop in ovo for different periods of time from 24 to $48 \mathrm{~h}$ depending on the experiment. The concentration of DNA above $6 \mu \mathrm{g} / \mathrm{ml}$ proved to be critical for the efficiency of the electroporation. Focal electroporations of HH24-25 otic vesicles were performed ex ovo, with the embryos immobilized over an agarose plate, following a procedure similar to the one described for HH20-21 stages. After electroporation, otic vesicles were dissected out from ectoderm and neural tube and cultured in DMEM supplemented with $10 \%$ Fetal Bovine Serum (BioWhittaker Europe), at $37^{\circ} \mathrm{C}$ in a watersaturated atmosphere containing $5 \% \mathrm{CO}_{2}$ as described previously (León et al., 1995; Pujades et al., 2006) for additional 12-20 h. Vectors used for electroporation were as follows: (1) pCIG-mId3-IRES-GFP (Elisa Martí, Institut de Biologia Molecular de Barcelona-Consejo Superior de Investigaciones Científicas, Barcelona, Spain), a vector carrying the full length of mouse Id3 gene (mId3), used to over-express Id3, (2) pCIG-actALK3IRES-GFP (T. Schultheiss, Harvard University, Cambridge, MA), a vector carrying a transgene that codes for the constitutively active form of the Bmp receptor ALK3, was used to activate the Bmp pathway, (3) pCIG-GFP vector, expressing a nuclear localized GFP protein was used in control experiments, and(4) BRE-tk-EGFP construct (Elisa Martí), was used to monitor Bmp signaling pathway activation at the transcriptional level. This vector contains a Bmp-responsive element (BRE) consisting of a multimerization of two distinct highly conserved sequences encompassing the genomic regions $-1105 / 1080$ and $-1052 /-1032$ of the mouse $I d 1$ promoter. This region contains the Smad binding elements that confer specificity to Bmp transcriptional activity dependent on Smad1,5,8 proteins (Korchynskyi and ten Dijke, 2002). BRE-tk-EGFP was co electroporated with pDsRed2 (Clontech) to monitor transfection efficiency.

Organotypic cultures of otic vesicles. Otic vesicles were dissected from E3.5-E4 embryos corresponding to stage $\mathrm{HH} 20-23$, transferred into four-well culture plates (Nunc) and incubated in DMEM at $37^{\circ} \mathrm{C}$ in a water-saturated atmosphere containing $5 \% \mathrm{CO}_{2}$ as described previously (León et al., 1995; Pujades et al., 2006) Additions were as follows: 1\% fetal bovine serum (BioWhittaker Europe), recombinant human Bmp4 (R\&D
Table 1. Oligonucleotide primers

\begin{tabular}{lll}
\hline Gene & 5' Sequence & 3' Sequence \\
\hline GAPDH & TTGGCATTGTGGAGGGTCTT & GTGGACGCTGGGATGATGTT \\
Atoh1 & AACCACGCCTTCGACCAG & TGCAGCGTCTCGTACTTGGA \\
Hes5 & GAAATCCTGACACCCAAAGAG & TCAATGCTGCTGTTAATCCT \\
Sox2 & AAGAGACCCTTCATTGAGGA & CGTGTACTTATCCTTCTTCATCAG \\
Lfg & GAAGAGCTGGGGAGGAAG & GCTCCACCATGAGCACCAG \\
Id 1 & GCACGGAGGGTCTCTAAAGT & CCAGCTGCAGGTCCCAGAT \\
Id2 & ACAGACATCAGCATCCTCTC & CACTCGCCATTAGTTCTGAG \\
Id3 & CCCACCCCACCATTATGA & GCCTCGTAACAGCTCCTGAC \\
\hline
\end{tabular}

Systems) at 20 and $100 \mathrm{ng} / \mathrm{ml}$, Noggin (R\&D Systems) at $1 \mu \mathrm{g} / \mathrm{ml}$ and Dorsomorphin (Biomol) at $10 \mu \mathrm{M}$.

Immunohistochemistry. Cryostat sections $(20 \mu \mathrm{m})$ of whole-mount ISH-treated, or naive embryos fixed overnight with $4 \%$ paraformaldehyde, were processed for immunohistochemistry as described previously (Pujades et al., 2006). Antibodies and dilutions used were as follows: $\alpha$-Jag1 rabbit polyclonal (Santa Cruz Biotechnology, Inc, 1:50); $\alpha$-Prox1 rabbit polyclonal (Abcam, 1:200); $\alpha$-MyoVIIa rabbit polyclonal (provided by T. Hasson, University of California San Diego, San Diego, CA, 1:5000); $\alpha$-GFP mouse monoclonal (Invitrogen, 1:400), $\alpha$-GFP rabbit polyclonal (Clontech, 1:400), $\alpha$-P-Smad1,5,8 rabbit polyclonal (Cell Signaling Technology, 1:200); $\alpha$-P-Smad1,5,8 rabbit polyclonal (provided by E. Laufer, Columbia University, New York, NY) (1:100), $\alpha$-Sox2 goat polyclonal (Santa Cruz Biotechnology, Inc, 1:400), $\alpha$-MyoVIIa mouse monoclonal (D. J. Orten, Boys Town National Research Hospital, Omaha, NE, 1:300) and $\alpha$-3A10 mouse monoclonal (T. Jessell, Columbia University, New York, NY, 1:600). Fluorescent detection was performed using Alexa-conjugated donkey secondary antibodies (Invitrogen, 1:400-1:2000). Detection of Sox2 antibody was also performed using HRP-conjugated-anti goat (Dako, 1:500), and developed with DAB subtract (Sigma).

Quantitation of results. To quantify changes in gene expression in the epithelium and in the mesenchyme separately, we adapted a method by (Matkowskyj et al., 2000). Briefly, several otic vesicles were serially sectioned and reconstructed (as shown in Fig. 2 D). Digitalized photomicrographs were acquired with the same conditions of magnification, illumination and capture settings and imported into ImageJ software (Rasband WS, ImageJ, U.S. National Institutes of Health, http://rsb.info. nih.gov/ij/, 1997-2008). An empty area situated at a position of the section not affected by the experimental treatment was used for reference and allowed comparison between different slides (Paizs et al., 2009). Images were converted to binary and set up an intensity threshold (control epithelia without ISH signal). Either the entire otic epithelium or the surrounding mesenchymal tissue were selected on each section, and measured above the threshold level for each compartment. At least 3 entire otic vesicles were processed in each condition (control, Bmp4, Noggin) and the results were plotted in a bar diagram where epithelial and mesenchymal area values of $I d 2$ expression are displayed in different columns (see Fig. 2E). The total area of epithelium (or mesenchyme) in each slide was considered as $100 \%$. Values are expressed as mean \pm SD. Quantification of Figure $3 G$ (see below) was done by counting cells from four equivalent electroporated domains that were double-labeled with MyoVIIa and GFP. The average fraction of MyoVIIa-positive hair cells expressing GFP after electroporation was plotted for $\mathrm{mId} 3$ and control electroporated regions.

Quantitative real-time PCR. Otic vesicles were isolated from $\mathrm{HH} 21-22$ embryos, and cultured either in control conditions or in the presence of different concentrations of Bmp4, Noggin or Dorsomorphin. RNA was isolated using RNeasy Mini kit (Qiagen) and purified mRNA was retrotranscribed with the Superscript III DNA polymerase (Invitrogen) using random primers (Invitrogen). Real time PCR was performed using SybrGreen master mix (Roche). For each quantitative real-time PCR (qRT-PCR) run, cDNA generated from 15 ng of RNA was used. Amplification was performed using LightCycler (Roche). Primer sets (Invitrogen) (Table 1) were designed to have comparable melt curves (melting temperature $=60^{\circ} \mathrm{C}$ ), and, when possible, spamming exon-exon junctions. GAPDH was used as calibrator gene. mRNA relative levels are the 
difference between each treatment and the control, calculated using the $\Delta \Delta \mathrm{Ct}$ method. Results are the mean of three PCR quantifications for each gene, for three independent retrotranscriptions, averaged from three different experiments (five otic vesicles per condition and experiment). Error bars represent SEM. One asterisk indicates $p<0.05$ and double asterisks $p<0.01$ levels of significance of the difference with respect to control values calculated by the Student's $t$ test.

\section{Results}

Id expression and Bmp activity in the prosensory patches of the otic vesicle The sensory organs of the inner ear are defined by the occurrence of differentiated hair cells, and they start to become apparent by E5 in the vestibular cristae of the chick embryo (Bartolami et al., 1991). However, several genes foreshadow the differentiation of the sensory organs and they define restricted epithelial domains that contain the sensory progenitors, thereby called prosensory patches (Adam et al., 1998). Bmp4 and 7 were the first genes shown to map to the prosensory patches (Oh et al., 1996; Wu and Oh, 1996), along with other genes like Serrate1 (Adam et al., 1998), Prox1 (Stone et al., 2003) and Sox2 (Neves et al., 2007). The first hair cells that differentiate are located in the cristae, where we concentrated our study (Bartolami et al., 1991; Oh et al., 1996; Wu and Oh, 1996; Fritzsch et al., 2002; Bell et al., 2008).

In the experiments that follow we analyzed the correspondence between the expression of $I d$ genes, Bmp ligands and Bmp activity readouts in the developing prosensory patches. Figure 1 shows the expression of Bmp4 and Bmp7 in the anterior prosensory patch of an E4 otocyst (Fig. $1 A, B$ ), and that of $I d 3$ (Fig. 1C). Sections were analyzed by ISH, and colabeled for Serrate1 by immunofluorescence (Fig. $1 D-F)$. The expression domains of Bmp4, $B m p 7$, and Id 3 overlapped extensively and contained the prosensory patches as defined by Serrate1. As shown, Bmp7 and $I d 3$, and to lesser extent Bmp4, extended also to the neighboring epithelium. The overlapping of Id expression domains and the prosensory patches is also illustrated in Figure 1G, where an E4 otocyst was probed for Id 2 by ISH and for the prosensory gene product Proxl by immunofluorescence (Fig. $1 G$, details in $H, I$ ). $I d 2$ was expressed along with Prox 1 in two poles of the otocyst that corresponded to the anterior and posterior prosensory patches of the E4 otocyst. Id 2 expression was also detected in the periotic mesenchyme adja-
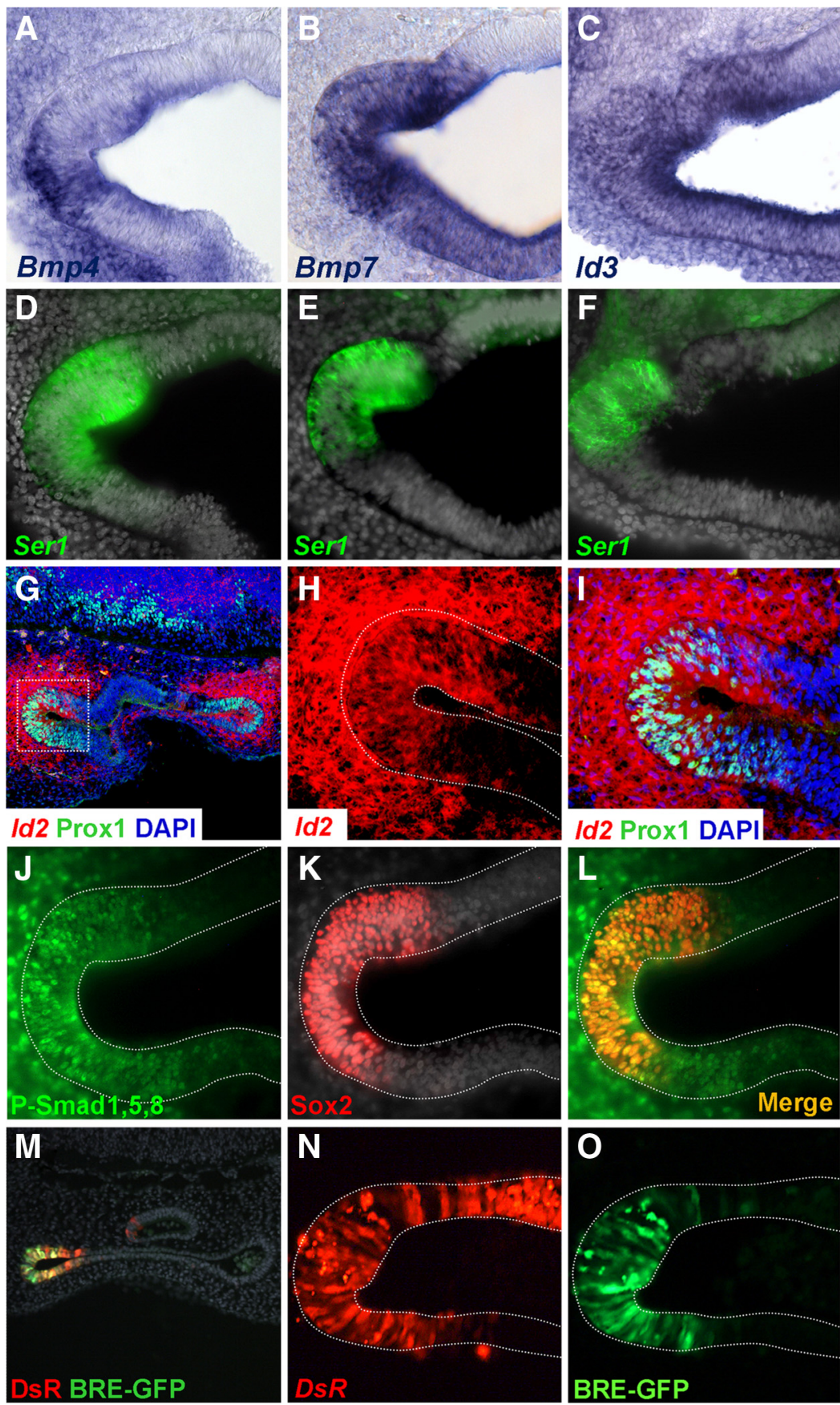

Figure 1. Bmp pathway and Id gene expression in the prosensory patches. Coronal sections of $\mathrm{E} 4$ embryos show prosensory domains of vestibular cristae. $\boldsymbol{A}-\boldsymbol{F}, B m p 4, B m p 7$, and Id3 expression in the prosensory patches. Otocysts were processed for ISH with Bmp4 (A), Bmp7 (B), or Id3 (C) riboprobes and for Serrate1 immunofluorescence (D-F). G-I, Id2 gene expression in the prosensory patches. Confocal images of coronal sections processed for ISH with Id2 probe (red) and for immunofluorescence for Prox1 (green). The in situ hybridization signal was captured by confocal microscopy in the near infrared, Id 2 was expressed in two epithelial regions corresponding to the prosensory domains of the cristae and the surrounding mesenchyme $(\boldsymbol{G})$. An enlargement of the boxed area is shown in $\boldsymbol{H}$ and $\boldsymbol{I}$ to better illustrate the superposition

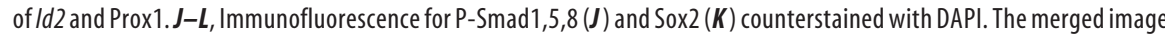
is shown in $L$. Note that P-Smad1,5,8 immunoreactivity overlaps the prosensory patch as identified by Sox2 expression, but it also extends outside the Sox2 domain into the neighboring epithelium and the surrounding mesenchyme. $\boldsymbol{M}-\mathbf{0}$, Smad transcriptional activity in prosensory patches. Cryostat section of the otic vesicle with the DsRed and the EGFP signal is shown in $\boldsymbol{M}$. The prosensory domain of the anterior crista exhibited DsRed $(\boldsymbol{N})$ and BRE directed EGFP signals $(\boldsymbol{O})$. Note that the extension of the DsRed signal is broader than the BRE directed EGFP signal that is restricted to the anterior pole of the otic vesicle. For better comparison, photographs from different preparations were flipped horizontally to show either the anterior or posterior cristae with the same orientation: $\mathbf{A}-\mathbf{F}, \mathbf{M - 0}$, anterior cristae; $\mathbf{G} \mathbf{L}$, posterior cristae; medial is always to the top. 
A
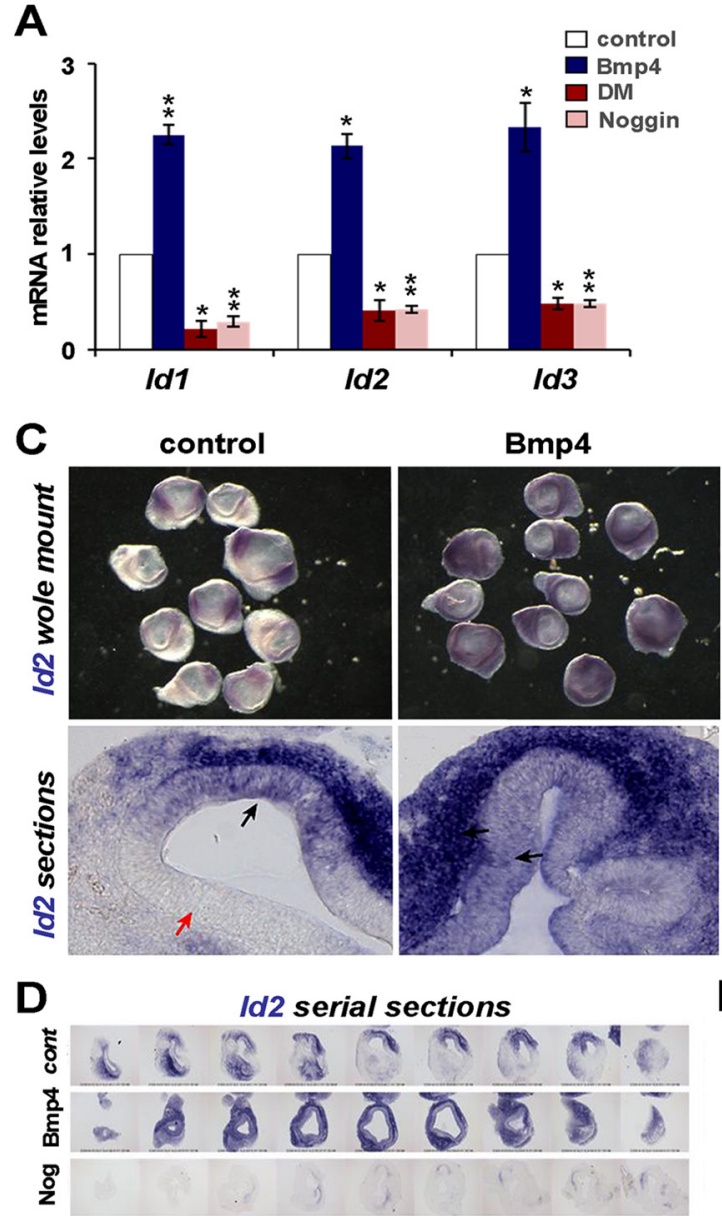

B
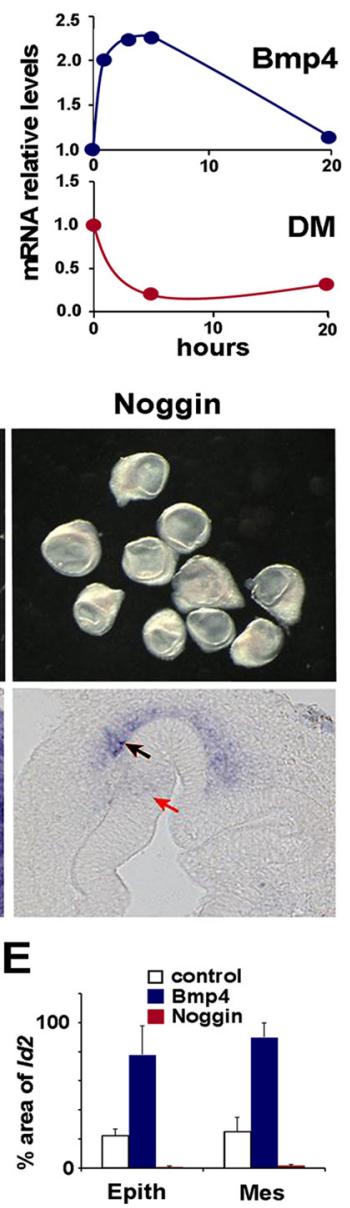

Figure 2. Effects of Bmp signaling on /d expression. A, Quantitative RT-PCR analysis of the effects on /d 1-3 mRNA levels after 5 h-incubation with Bmp4 (20 ng/ml, blue), Dorsomorphin (10 $\mu \mathrm{m}$, red), or Noggin (1 $\mu \mathrm{m}$, light red). mRNA levels in treated vesicles (blue, red and light red bars) are referred to control values (white bars), which were arbitrarily set to 1 and normalized for GAPDH expression. Error bars represent SEM. One asterisk indicates $p<0.05$ and double asterisks $p<0.01$ levels of significance of the difference with respect to control values calculated by the Student's $t$ test. All three Id genes were induced with Bmp 4 treatment and downregulated by Bmp blockade with either Noggin or Dorsomorphin. B, Time course of $I d 1$ expression after treatment with Bmp4 (20 ng/ml, top graph), or Dorsomorphin ( $10 \mu \mathrm{m}$, bottom graph). C, Effect of Bmp4 on /d2 expression in situ. Otic vesicles were isolated at HH21 and cultured for $4 \mathrm{~h}$ either in control media, or with the addition of $100 \mathrm{ng} / \mathrm{ml} \mathrm{Bmp} 4$, or $1 \mu \mathrm{g} / \mathrm{ml}$ Noggin. The top row shows whole-mount otic vesicles and the bottom row cryostat sections of the cultured otic vesicles. Black arrows indicate sites of strong / 22 expression and red arrows indicate sites where $/ d 2$ was not detected. $D$, Serial sections covering an entire otic vesicle, showing one example for each condition. $\boldsymbol{E}$, Quantitative analysis of the effects of Bmp4 and Noggin on Id2 expression as evaluated by ISH. Values of the columns represent the percentage of surface area positive for Id2-positive signal in both epithelium and mesenchyme (see Materials and Methods).

cent to the sensory patches. This pattern of expression was similar for Id1-3 (Fig. 1, compare $C, H$ ) (data not shown).

The main intracellular pathway involved in the response to Bmps is the phosphorylation of Smad1,5,8 proteins, which results in binding to Smad4, the translocation of the complex to the nucleus and the regulation of gene expression (Massagué et al., 2005). Figure $1 J-L$ shows that the domains of Bmp expression exhibit also high levels of Bmp activity. This is illustrated by the immunodetection of the phosphorylated form of Smad1,5,8 (a common P-Smad1,5,8 epitope) in the prosensory domains of the inner ear of an E4 otocysts. The levels of P-Smad1,5,8 were high in the prosensory patch, where it colocalized with Sox2 (Fig. $1 \mathrm{~J}, L$ ). Like $I d s$, P-Smad1,5,8 extended outside the Sox2-positive domain and also to the surrounding mesenchyme (Fig. $1 \mathrm{~L}$ ), indicating that those were also domains of high Bmp activity.

To analyze the relationship between Bmp activity and Id expression, we used a Bmp activity reporter that contains the Bmp-

Responding Element (BRE) derived from a multimerization of specific sequence elements of the mouse Id1 promoter (Korchynskyi and ten Dijke, 2002). The BRE-tk-EGFP reporter provides a readout of the ability of the Bmp/Smad pathway to activate Id expression. BRE-tk-EGFP was coelectroporated with pDsRed as a control for transfection, in E3 otic vesicles, and their expression detected after $24 \mathrm{~h}$ by direct fluorescence (Fig. 1M-O). EGFP expression driven by BRE was detected in the prosensory domains, at the anterior and posterior poles of the $\mathrm{E} 4$ otic vesicle (Fig. $1 M$ ) (data not shown). Note that in the example shown in Figure $1 M-O$, the expression of EGFP was restricted to the anterior pole and that it was narrower than the DsRed expression domain (Fig. 1, compare $N, O$ ). The EGFP patch corresponded well to the Id and the P-Smad1,5,8positive domains described above. These experiments indicate that the endogenous levels of Bmp activity in the prosensory patches are sufficient to activate the Bmp responsive element of the Id 1 gene promoter.

In summary, the results show that the prosensory patches of the developing otocyst are contained within domains of high levels of Bmp4 and 7 mRNA expression and P-Smad1,5,8 accumulation. Bmp signaling within these domains corresponds faithfully with a strong promoter activity and mRNA expression of Id genes.

\section{Bmp signaling regulates $I d$ expression during prosensory development}

The above experiments suggest that Bmp signaling regulates the expression of $I d$ genes in the prosensory patches. To further analyze this question, we studied the effects of enhanced Bmp activity or Bmp blockade on Id expression. Otic vesicles from E3 (HH20) embryos were isolated by microsurgery, grown in culture during $24 \mathrm{~h}$, and then analyzed for gene expression by qRT-PCR, using specific oligonucleotide primers (Table 1). Incubation medium contained recombinant hBmp4 $(20 \mathrm{ng} / \mathrm{ml})$ as an activator of the Bmp signaling pathway, and either Dorsomorphin (DM, $10 \mu \mathrm{M})$ or Noggin $(1 \mu \mathrm{g} / \mathrm{ml})$ as inhibitors (Balemans and Van Hul, 2002; Anderson and Darshan, 2008). Bmp4 induced the expression of Id1-3 (Fig. $2 \mathrm{~A}$, blue bars). The increase in mRNA levels was $\sim 2.5$ fold for Bmp4 $20 \mathrm{ng} / \mathrm{ml}$, and $\sim 5$-fold for $100 \mathrm{ng} / \mathrm{ml}$ (data not shown). The induction of Id genes by Bmp4 was rapid, levels of Id1-3 transcripts increasing already after $1 \mathrm{~h}$ of incubation (Fig. $2 B$, top diagram). The addition of Dorsomorphin $(10 \mu \mathrm{M})$ or Noggin $(1 \mu \mathrm{g} / \mathrm{ml})$ resulted in a strong and also rapid reduction of endogenous $I d$ expression (Fig. $2 A$, red and light red bars; $B$, bottom diagram). Therefore, either the blockade of Bmpreceptor interaction with Noggin or receptor phosphorylation with Dorsomorphin reduced the levels of Id1-3 mRNAs, suggest- 
ing that at this developmental stage, the expression of $I d s$ in the otic vesicle is strictly dependent on Bmp activity described above.

Since Id genes were expressed both in the epithelium and its surrounding mesenchyme, we used in situ hybridization to analyze further the spatial distribution of the changes in Id expression upon changes in Bmp activity. A typical experiment is shown in Figure $2 C$ for $I d 2$. In control medium, $I d 2$ expression was restricted to anterior and posterior poles of the otic vesicle, which correspond to the normal expression domains described in situ (Fig. $1 G$; compare Fig. $2 C$, control, with the in vivo expression pattern shown). Bmp4 treatment $(5 \mathrm{~h})$ produced a strong and ubiquitous induction of $I d 2$ expression (Fig. $2 C$; Bmp $4, n=10 / 10,4$ different experiments). On the contrary, the incubation with Noggin during the same period abolished Id2 expression (Fig. 2C; Noggin, $n=10 / 10,4$ experiments). This is important because it indicates that at this stage of development, the epithelial expression of $I d$ is strictly dependent on the endogenous Bmp signaling. Similar results were obtained for Id 1 and $\operatorname{Id} 3$ ( $n=$ $10 / 10$, at least two experiments for each gene, data not shown). Cryostat sections of cultured otic vesicles showed that Id expression was upregulated by Bmp both in the otic epithelium and in the surrounding mesenchyme (Fig. 2C; Bmp4, bottom row). Conversely, after incubation with Noggin, only a few vesicles $(n=2 / 10)$ retained a weak residual signal for $I d 2$, and this was mostly located in the adjacent mesenchyme (Fig. 2C; Noggin, bottom row, black arrows) The quantification of the expression in the epithelium and in the mesenchyme is shown in the bar diagram of Figure $2 E$ (raw data from serial sections are shown in $D$ ) (see Materials and Methods). The effects of Bmp on Id expression was paralleled by the phosphorylation of Smad1,5,8 (supplemental Fig. $1 A$, available at www.jneurosci.org as supplemental material). The activation of the Bmp pathway in vivo, by electroporation of the active form of the active Alk3 Bmpreceptor also induced $I d$ expression (supplemental Fig. $1 \mathrm{~B}$, available at www.jneurosci.org as supplemental material).

\section{Id3 inhibits Atoh1 expression}

As mentioned above, Bmp signaling prevents Atoh1 expression and the generation of hair cells in the chick otic vesicle (Pujades et al., 2006). Given that Bmps regulate $I d$ expression, one interesting possibility is that $I d$ s mediate this effect by interfering with Atoh 1 expression. With this in mind, we studied whether Ids were sufficient to prevent Atoh1 expression and hair cell differentiation. Otic vesicles (HH20) were electroporated in ovo with a vector expressing $\mathrm{mId} 3$ and analyzed after for Atoh1 and MyoVIIa expression. Atoh1 is a master gene for hair cell determination (Bermingham et al., 1999; Zheng and Gao, 2000), and MyoVIIa is an early marker of hair cell differentiation (Sahly et al., 1997). Electroporations were performed focally, in the anterior aspect of the otic vesicle, targeting the prospective anterior crista (Fig. $3 A-C$ ). Cells overexpressing $\mathrm{mId} 3 \mathrm{did}$ not express Atoh 1 or MyoVIIa and concentrated in the basal aspect of the epithelium (Fig. 3D,E, respectively). On the contrary, cells that were electroporated with a control GFP vector showed an even distribution across the epithelium, and they indistinctively expressed MyoVIIa (Fig. $3 F$ ). Note that outside the sensory region, mId3-positive cells resided throughout the depth of the epithelium, but within the sensory patch they remained concentrated at its basal aspect (Fig. 3, compare D, E). Quantification of the results was done by counting cells from equivalent electroporated domains that were double-labeled with MyoVIIa and GFP. The fraction of MyoVIIa-positive hair cells expressing GFP after $\mathrm{mI} 33$ electroporation was much reduced when compared with the control electroporation (Fig. 3G). This indicates that mId3 either maintains cells in the progenitor state, it directs supporting versus hair cell fate, or both. 
E5.5
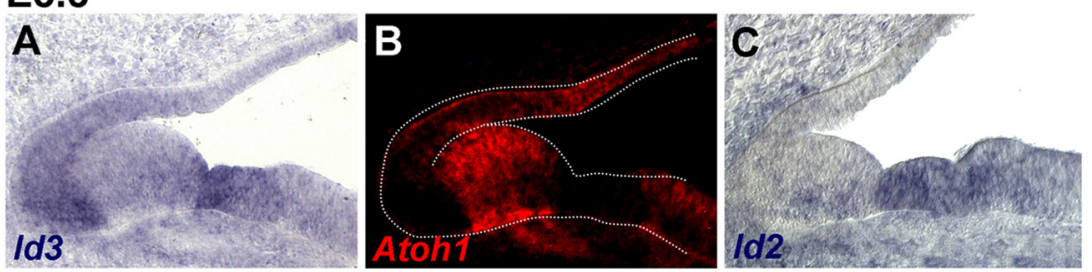

E7
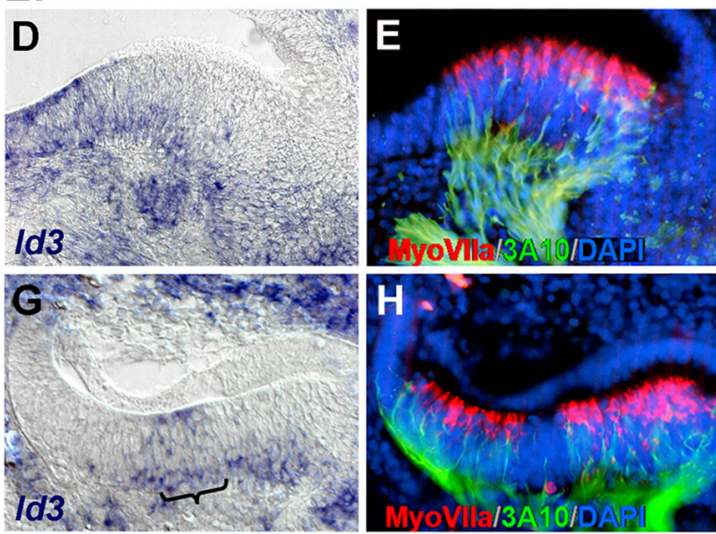

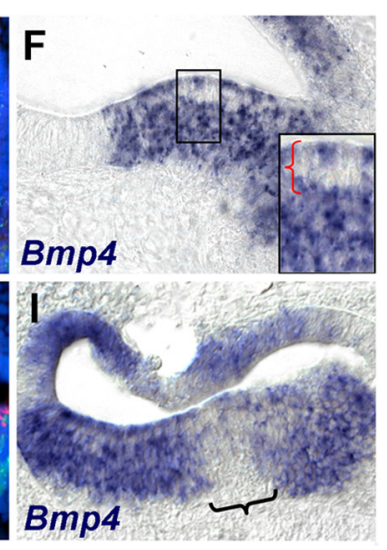

Figure 4. Bmp and Id expression during differentiation of the sensory patches. $\boldsymbol{A}-\boldsymbol{C}$, Transversal alternate sections of the inner ear of E5.5 embryos, at the level of the lateral crista, double-labeled for Id3 (A) and Atoh 1 ( $\boldsymbol{B})$ by fluorescent double ISH, and for Id 2 (C). Atoh1 and Id3 were expressed complementary to each other. D-I, Transversal alternate sections of the inner ear of E7 embry0s, at the level of the lateral $(\boldsymbol{D}-\boldsymbol{F})$ and posterior $(\boldsymbol{G}-\boldsymbol{I})$ cristae, probed for Id3 $(\boldsymbol{D}, \boldsymbol{G})$ and Bmp4 $(\boldsymbol{F}, \boldsymbol{I})$ by ISH. The brackets in $\boldsymbol{G}$ and $\boldsymbol{I}$ indicate the expression of $/ d 3$ in nonsensory epithelium devoid of $B m p 4$ signal, the cruciatum. The box in $\boldsymbol{F}$ shows a detail of a region containing a row of hair cells (red bracket), with reduced or absent Bmp4 signal. $\boldsymbol{E}$ and $\boldsymbol{H}$ are the same as $\boldsymbol{D}$ and $\boldsymbol{G}$, which were colabeled with MyoVIla (red) and 3A10 (green) antibodies to better identify the sensory domain and the developing hair cells. Nuclei were counterstained with DAPI (blue).

The effect of mId3 on Atoh1 expression was also analyzed by qRT-PCR. Otic vesicles were electroporated as described above and, after $24 \mathrm{~h}$, the targeted domain was dissected for qRT-PCR analysis (Fig. $3 H$ ). The results show that $\mathrm{mId} 3$ was sufficient to reduce the levels of Atoh1 mRNA (Fig. $3 H$, left). The downregulation of Atoh 1 by $\mathrm{mId} 3$ was paralleled by the reduced expression of Hes5 (Fig. 3H), as expected from the concomitant decrease of lateral inhibition (see below). Prosensory genes like Sox 2 or Lfng remained unaffected by $\mathrm{m} I d 3$ overexpression (Fig. $3 H$ ), suggesting that the effect of mId3 occurred at the level of Atoh 1 expression and hair cell determination and not of prosensory specification. The reduction in Atoh1 expression was not associated with cell death as judged from caspase- 3 immunodetection (data not shown).

The effects of mId3 on Atoh1 mimic those of Bmp4 (supplemental Fig. 2, available at www.jneurosci.org as supplemental material). Atoh 1 mRNA levels were reduced by Bmp4 and increased after incubation with Dorsomorphin. In parallel, Hes5 expression was also induced by Dorsomorphin and reduced by Bmp4 (supplemental Fig. $2 B$, available at www.jneurosci.org as supplemental material), suggesting that it was the result of lateral inhibition. In agreement, the induced expression of Hes5 was dependent on Notch activation as shown by its blockade by the $\gamma$-secretase inhibitor DAPT (supplemental Fig. 2C, available at www.jneurosci.org as supplemental material).

Together, the results show that $I d$ genes are expressed in the prosensory patches, they are regulated by the Bmp signaling pathway, and their overexpression mimic the inhibitory effect of Bmps on Atoh1 expression and hair cell differentiation. This suggests that one function of $I d s$ in the prosensory domains is to

prevent Atoh1 expression and the generation of hair cells, which would require the downregulation of the Bmp pathway.

Id genes are downregulated during hair cell differentiation

To test the relationship between $I d$ expression and hair cell generation, we analyzed Id1-3 expression during hair cell differentiation, and compared it with $B m p$ expression and activity. The analysis of Atoh 1 and Id 3 by double ISH hybridization showed that the expression of $I d$ genes was downregulated in the regions that initiated Atoh1 expression. This mutually exclusive pattern is illustrated by the sections of E5.5 (HH28) otocysts shown in Figure $4, A$ and $B$, where $I d 3$ expression was reduced in the lateral crista and concentrated at the boundaries and the surrounding nonsensory epithelium (Fig. 4A). Similar results were obtained for $I d 2$ as illustrated by probing the alternate sections (Fig. 4C).

By E7, Ids were mostly absent from the cristae, their expression remaining high at the edges of the sensory epithelium and in the neighboring nonsensory regions. This is illustrated in Figure 4, D and $G$, which show sections of E7 lateral and posterior cristae that were probed for $I d 3$, and counterstained with MyoVIIa and 3A10 antibodies (Fig. 4E,H), which label newborn hair cells and innervating otic neurons, respectively (Sahly et al., 1997; Sánchez-Calderón et al., 2007). Id expression was very much reduced or absent within the domains where hair cells differentiated (Fig. 4, compare $D, E$, and $G, H$ ). The expression of $B m p 4$ was also reduced from hair cells as shown by probing the alternate sections (Fig. 4F,I). Note the white row of cells in the luminal aspect of the sensory epithelium in Figure $4 F$ which was boxed and enlarged (Fig. $4 F$, inset; red brackets show the level of the row of hair cells) (see also Oh et al., 1996). The expression of $B m p 4$, however, was strong in other cells within the sensory domain, where $I d s$ were not expressed anymore (Fig. 4, compare $G$, I). Bmp7 was also expressed in the cristae and the surrounding epithelium (Wu and Oh, 1996) (data not shown).

\section{Bmp activity during hair cell differentiation}

The above experiments pose two interrelated questions: first, what is the relationship between Bmp expression and Bmp activity in the differentiating sensory organs and, second, what is the relationship between Bmp activity and hair cell differentiation. With this in mind, we analyzed the Bmp-dependent P-Smad1,5,8 pathway in the vestibular cristae of E5 and E7 embryos (Fig. 5). Immunoreactivity against P-Smad1,5,8 (Fig. 5A,D) is shown along with MyoVIIa (Fig. $5 B, E$ ) and Sox 2 (Fig. $5 C, F$ ) to identify hair cells and the sensory domain, respectively. In E5, most cells of the sensory cristae were positive for P-Smad1,5,8 except a few hair cells (Fig. 5A-C; see an enlarged detail of hair cells from boxed regions). By E7, P-Smad1,5,8 signal was still high in the basal layer of the sensory patch (Fig. 5D), but it was absent from most hair cells (Fig. 5 D,E). Taking P-Smad 1,5,8 phosphorylation as a readout of the activation of the Bmp pathway, the results 
indicate that Bmp activity is switched off in nascent hair cells. This shows that there is a good correspondence between the downregulation of Bmp expression in hair cells, the loss of Bmp activity and the downregulation of $I d s$ during the differentiation of hair cells (Fig. 4D,F).

As noted above, most cells of the sensory patch other than hair cells expressed Bmp4 and exhibited high P-Smad1,5,8. However, the expression of Ids in the supporting cell layer of the sensory patch was very low or absent. This is somehow paradoxical because as shown above, the link between Bmp activity and Id expression was strong during prosensory development. Because the levels of P-Smad1,5,8 were high in the sensory patch, the downregulation of Id expression from supporting cells ought to be caused by mechanisms that operate either downstream of Smad1,5,8 phosphorylation or that are independent of the $\mathrm{Bmp} / \mathrm{Smad}$ pathway. To analyze this question, we made use of the Bmp reporter described previously. Otic vesicles of E4.5 embryos were coelectroporated with BRE-tk-EGFP and pDsRed, and allowed to develop for $24 \mathrm{~h}$ in vitro, to an equivalent stage of E5.5. Electroporation was targeted to the anterior crista to test the Smad transcriptional activity within the sensory patch. The embryos were then sectioned and processed by immunofluorescence for Sox 2 expression to identify the sensory patches (Fig. $6)$. The results show that BRE-directed EGFP expression was indeed detected in the supporting cells of the sensory patches (Fig. $6 B, E$ ), indicating that those cells were able to activate endogenously the Smad-dependent regulatory region of the Idl gene. This suggests that the lack of expression of $I d s$ in the supporting cells described above most probably depends on other factors that regulate the transcription or the stability of $I d$ mRNAs, with independence of the Bmp/Smad pathway.

\section{Discussion}

The experiments described in this paper were aimed at studying the mechanisms by which Bmp signaling regulates the development of hair cells in the inner ear. In particular, we focused on the regulation and function of $I d$ genes as potential mediators of Bmp effects on Atoh1 expression and hair cell generation.

\section{Id genes regulate Atoh 1 expression and the generation of hair cells}

The generation of hair cells requires the activity of the bHLH factor Atoh1 (Bermingham et al., 1999; Zheng and Gao, 2000), but the factors that regulate Atoh1 transcription during inner ear development are largely unknown. Experiments on isolated otic vesicles showed that Bmp4 inhibits the expression of Atohl in the prosensory patches and the blockade of Bmp activity enhances Atoh1 expression and hair cell generation (Pujades et al., 2006) (present work). This is reminiscent of the function of Bmp signals
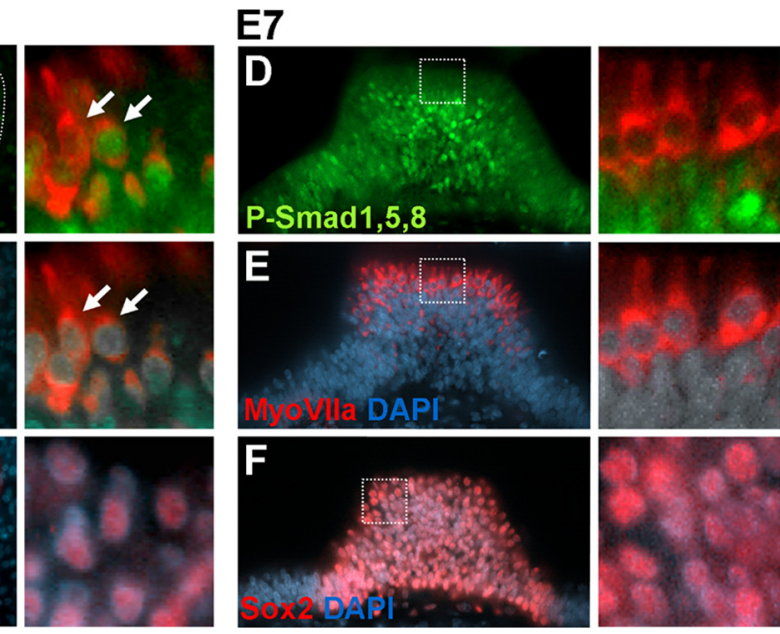

Figure 5. P-Smad1,5,8 in sensory organs. $\boldsymbol{A}-\boldsymbol{C}$, Coronal section of $\mathrm{E} 5$ otocysts showing the anterior crista colabeled for P-Smad1,5,8 ( $\boldsymbol{A}$, green) and MyoVIla ( $\boldsymbol{B}$, red). The corresponding alternate serial section was stained for Sox2 ( $\boldsymbol{C}$, red). Sections were counterstained with DAPI (blue). The details show the double-labeling with MyoVIla and P-Smad1,5,8 and MyoVIla and DAPI. in , of comparison (detail of C). D-F, Coronal section of E7 otocysts showing the posterior crista colabeled for P-Smad1,5,8 (D,
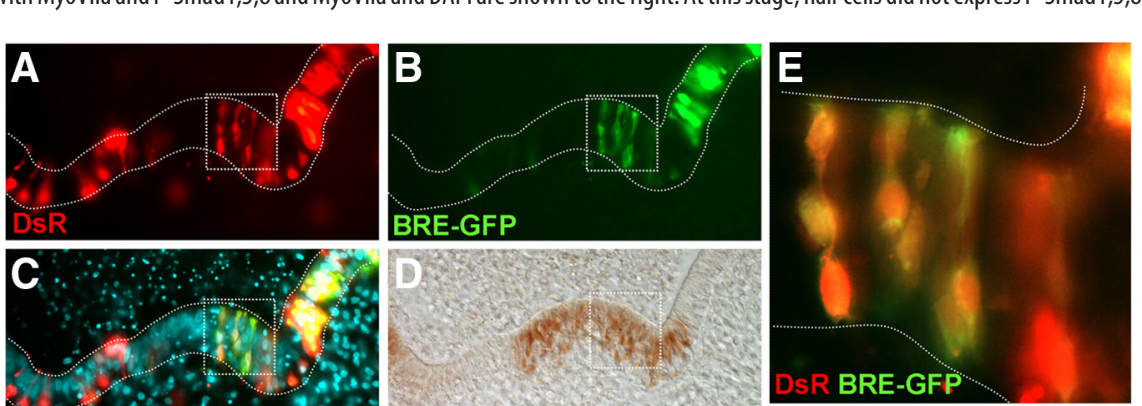

Sox2

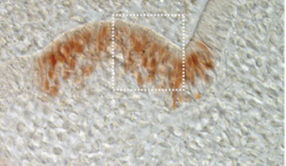

BRE-GFP

Figure 6. Smad transcriptional activity in differentiating sensory organs. Smad transcriptional activity was monitored with BRE-tkimage is shown in $\boldsymbol{C}$. The same section was immunostained for Sox2 $(\boldsymbol{D})$ to identify the location of the sensory patches. The boxed area in $\boldsymbol{A}-\boldsymbol{D}$ was magnified in $\boldsymbol{E}$, showing the overlapped signal of DsRed and BRE-EGFP within the Sox2-positive domain.

in different ES cells and neural progenitors, where Bmps block neuronal differentiation and maintain cells in an undifferentiated state (Varga and Wrana, 2005; Chen and Panchision, 2007), and where Bmps oppose Atoh1 and other proneural genes (Bertrand et al., 2002; Grimmer and Weiss, 2008; Zhao et al., 2008). The effects of Bmp on Atoh1 and hair cell development would be an extension of this general function of Bmps to prevent the premature differentiation of an already neural committed cell population. Ids are Bmp-induced-immediate early genes in ES cells, where they also function as inhibitors of cell differentiation through the inactivation of bHLH transcription factors (Hollnagel et al., 1999; Yokota, 2001). In this work we demonstrate that $I d s$ are expressed in the prosensory patches before hair cell differentiation along with Bmp4 and Bmp7. Those are domains of high Bmp activity as judged by the accumulation of the phosphorylated form of Bmp-dependent R-Smad (P-Smad1,5,8).Bmp signaling regulates $I d$ expression in the prosensory patches and $I d 3$ is sufficient to inhibit Atoh1 expression and hair cell differentiation. This suggests that $I d s$ mediates the function of Bmp signaling in preventing hair cell differentiation. It is also likely, however, that Atohl expression is under the control of other factors and signals that are expressed in the prosensory domains. How these factors 
interact to precisely time the initiation of hair cell differentiation remains to be elucidated.

Id proteins act primarily as dominant-negative regulators of tissue-specific bHLH transcription factors and prevent them from forming functional complexes with transcriptional activity (Yokota, 2001). This mechanism cannot account for the reduction in Atoh1 mRNA levels after Id overexpression shown in the present work, neither for the observation that E47 overexpression does not block Id function (Jones et al., 2006). However, it is known that Ids also increase the degradation rate of bHLH factors (Viñals et al., 2004; Viñals and Ventura, 2004). The Atoh1 gene contains an E-box consensus binding site in its enhancer region that is related to its autoregulation (Helms et al., 2000), this enhancer being active in developing hair cells (Woods et al., 2004). Hence, the Id-induced destabilization of Atoh1 may result in the negative regulation of Atoh1 transcription (Zhao et al., 2008). In summary, BMP-mediated Id expression within the prosensory patches, may function as a molecular switch by functionally blocking the expression and the activity of Atoh1.

\section{Id genes are regulated by Bmp in the prosensory patches}

The regulation of $I d$ genes by Bmp has been extensively studied in several model systems (Yokota, 2001; Ruzinova and Benezra, 2003), but it had not been previously analyzed in the inner ear. We show that $I d 1-3$ genes are induced in response to the activation of the Bmp pathway. The initiation of the response is very fast, in agreement with Id1-3 being targets of Bmp, as shown in other model systems (Hollnagel et al., 1999). Bmp blockade, with either Noggin or Dorsomorphin, rapidly reduces Id expression in the prosensory epithelium, indicating that the endogenous Bmp signaling is required to maintain $I d$ expression in vivo. This is consistent with the fast turnover of the Id mRNA and protein which have reported half-lives of 20-60 min, depending on the cell type (Deed et al., 1996; Bounpheng et al., 1999; Norton, 2000). Dorsomorphin specifically blocks the phosphorylation of R-Smad mediated by type I Bmp-receptors without affecting other signaling pathways that can be activated by Bmps (Anderson and Darshan, 2008; Cuny et al., 2008; Hao et al., 2008; Yu et al., 2008). This suggests that $I d$ expression in the prosensory domains of the early otocyst depends strictly on the steady activation of the Bmpinduced Smad signaling pathway. Accordingly, Id1-3 are expressed in the otic vesicle in broad regions that include the prosensory patches, and that map to the domains of Bmp4 and Bmp7 expression (Oh et al., 1996; Cole et al., 2000). These domains also coincide with those of high P-Smad1,5,8 immunoreactivity and BRE-tk-EGFP activity (present work). Therefore, in the prosensory patches there is a good correspondence between the regulation and function of the Bmp pathway and that of Id genes.

\section{The regulation of $I d s$ during hair cell differentiation}

Although $I d 1-3$ are expressed in the prosensory patches of the otic vesicle, further in development, $I d$ s are downregulated from hair cells and remain weakly expressed in basal layers of the sensory domains. The withdrawal of Id expression from hair-cells parallels the downregulation of Bmp4 and the loss of P-Smad1,5,8. The loss of $B m p 4$ in hair cells of the sensory organs was already noted by Oh et al. (1996) and confirmed here. The loss of Id expression from nascent hair cell would release the inhibition on Atoh1 expression and allow their differentiation. We like to suggest that the prosensory function of Bmp relies on the regulation of Id genes in the prosensory domains, which, in turn, maintain the undifferentiated state of prosensory progenitors and the inhibition of Atoh1 expression. Further in development, Bmp ligands are downregulated from nascent hair cell with the concomitant silencing of the Bmp/Smad pathway and Id expression. This, along with other factors, would allow the initiation of the differentiation of hair cells.

\section{The differential regulation of $I d s$ and the multiple functions of Bmp signaling in ear development}

During differentiation of the sensory patches, Id gene expression concentrates at the edges of the sensory regions, but it is downregulated from the supporting cell layer. Both are domains of high P-Smad1,5,8 activity: sensory patches express Bmp4 and the boundaries and surrounding nonsensory domains express high levels of Bmp7 (Oh et al., 1996). Why are Ids silenced in the supporting cell layer of the sensory patches, and not from the neighboring cells? The use of the Bmp/Smad transcriptional reporter BRE-tk-EGFP indicates that the Bmp/Smad pathway is transcriptionally active on the $I d$ promoter region, both within and at the edges of the sensory patches, suggesting that other factors block the expression of $I d s$ in the supporting cell layer. The nature of these factors remain unknown. It is possible that the balance between Bmp/Smad activity and other signals may dictate the final output, or that it depends on the specific combination of different Bmps, all possibilities remaining unexplored.

Bmps show multiple and seemingly paradoxical effects in neural development (Chen and Panchision, 2007). For ear development, Chang et al. (2008) had proposed a model where a sequential action of Bmp4 is required first for prosensory development, and second for the specification of supporting cells and the nonsensory elements of the sensory organs. Chang et al. (2008) suggest that persisting Bmp signaling in the supporting cell layer and in the edges of the patches contribute to specify supporting and nonsensory cell fates respectively. Supporting cells are under the effects of Bmp signaling and they express Bmptarget genes like Msxl and Lmo4 (Chang et al., 2008), but they exhibit low Id expression. This situation may allow Bmps to carry out functions during cellular differentiation which would be otherwise hampered by the inhibition of differentiation exerted by $I d s$. Contrarily, the boundaries of the sensory domains show high levels of P-Smad1,5,8, they express Bmp7 (Wu and Oh, 1996) (data not shown), and Bmp targets like Id genes (present work), Gata3, Lmo4 and p75, but not Msx1 (Chang et al., 2008). This would maintain the boundaries of the sensory organs in a proliferative and uncommitted state until later stages of development. In summary, the coupling and uncoupling between Bmp activity and Id expression may provide a mechanism for the diverse and seemingly opposing functions of Bmps throughout development.

\section{References}

Abello G, Alsina B (2007) Establishment of a proneural field in the inner ear. Int J Dev Biol 51:483-493.

Adam J, Myat A, Le Roux I, Eddison M, Henrique D, Ish-Horowicz D, Lewis J (1998) Cell fate choices and the expression of Notch, Delta and Serrate homologues in the chick inner ear: parallels with Drosophila sense-organ development. Development 125:4645-4654.

Anderson GJ, Darshan D (2008) Small-molecule dissection of BMP signaling. Nat Chem Biol 4:15-16.

Balemans W, Van Hul W (2002) Extracellular regulation of BMP signaling in vertebrates: a cocktail of modulators. Dev Biol 250:231-250.

Bartolami S, Goodyear R, Richardson G (1991) Appearance and distribution of the $275 \mathrm{kD}$ hair-cell antigen during development of the avian inner ear. J Comp Neurol 314:777-788.

Bell D, Streit A, Gorospe I, Varela-Nieto I, Alsina B, Giraldez F (2008) Spatial and temporal segregation of auditory and vestibular neurons in the otic placode. Dev Biol 322:109-120.

Benezra R, Davis RL, Lassar A, Tapscott S, Thayer M, Lockshon D, Weintraub H (1990) Id: a negative regulator of helix-loop-helix DNA binding pro- 
teins. Control of terminal myogenic differentiation. Ann N Y Acad Sci 599:1-11.

Bermingham NA, Hassan BA, Price SD, Vollrath MA, Ben-Arie N, Eatock RA, Bellen HJ, Lysakowski A, Zoghbi HY (1999) Math1: an essential gene for the generation of inner ear hair cells. Science 284:1837-1841.

Bertrand N, Castro DS, Guillemot F (2002) Proneural genes and the specification of neural cell types. Nat Rev Neurosci 3:517-530.

Bounpheng MA, Dimas JJ, Dodds SG, Christy BA (1999) Degradation of Id proteins by the ubiquitin-proteasome pathway. FASEB J 13:2257-2264.

Chang W, Nunes FD, De Jesus-Escobar JM, Harland R, Wu DK (1999) Ectopic noggin blocks sensory and nonsensory organ morphogenesis in the chicken inner ear. Dev Biol 216:369-381.

Chang W, ten Dijke P, Wu DK (2002) BMP pathways are involved in otic capsule formation and epithelial-mesenchymal signaling in the developing chicken inner ear. Dev Biol 251:380-394.

Chang W, Lin Z, Kulessa H, Hebert J, Hogan BL, Wu DK (2008) Bmp4 is essential for the formation of the vestibular apparatus that detects angular head movements. PLoS Genet 4:e1000050.

Chen HL, Panchision DM (2007) Concise review: bone morphogenetic protein pleiotropism in neural stem cells and their derivatives-alternative pathways, convergent signals. Stem Cells 25:63-68.

Cole LK, Le Roux I, Nunes F, Laufer E, Lewis J, Wu DK (2000) Sensory organ generation in the chicken inner ear: contributions of bone morphogenetic protein 4, serrate1, and lunatic fringe. J Comp Neurol 424: 509-520.

Cuny GD, Yu PB, Laha JK, Xing X, Liu JF, Lai CS, Deng DY, Sachidanandan C, Bloch KD, Peterson RT (2008) Structure-activity relationship study of bone morphogenetic protein (BMP) signaling inhibitors. Bioorg Med Chem Lett 18:4388-4392.

Deed RW, Armitage S, Norton JD (1996) Nuclear localization and regulation of Id protein through an E protein-mediated chaperone mechanism. J Biol Chem 271:23603-23606.

Elisa Piedra M, Borja Rivero F, Fernandez-Teran M, Ros MA (2000) Pattern formation and regulation of gene expressions in chick recombinant limbs. Mech Dev 90:167-179.

Fritzsch B, Beisel KW, Jones K, Fariñas I, Maklad A, Lee J, Reichardt LF (2002) Development and evolution of inner ear sensory epithelia and their innervation. J Neurobiol 53:143-156.

Fritzsch B, Beisel KW, Hansen LA (2006) The molecular basis of neurosensory cell formation in ear development: a blueprint for hair cell and sensory neuron regeneration? Bioessays 28:1181-1193.

Gerlach LM, Hutson MR, Germiller JA, Nguyen-Luu D, Victor JC, Barald KF (2000) Addition of the BMP4 antagonist, noggin, disrupts avian inner ear development. Development 127:45-54.

Grimmer MR, Weiss WA (2008) BMPs oppose Math1 in cerebellar development and in medulloblastoma. Genes Dev 22:693-699.

Hamburger V, Hamilton HL (1992) A series of normal stages in the development of the chick embryo. 1951. Dev Dyn 195:231-272.

Hao J, Daleo MA, Murphy CK, Yu PB, Ho JN, Hu J, Peterson RT, Hatzopoulos AK, Hong CC (2008) Dorsomorphin, a selective small molecule inhibitor of BMP signaling, promotes cardiomyogenesis in embryonic stem cells. PLoS One 3:e2904.

Helms AW, Abney AL, Ben-Arie N, Zoghbi HY, Johnson JE (2000) Autoregulation and multiple enhancers control Math1 expression in the developing nervous system. Development 127:1185-1196.

Hogan BL (1996) Bone morphogenetic proteins in development. Curr Opin Genet Dev 6:432-438.

Hollnagel A, Oehlmann V, Heymer J, Rüther U, Nordheim A (1999) Id genes are direct targets of bone morphogenetic protein induction in embryonic stem cells. J Biol Chem 274:19838-19845.

Jones JM, Montcouquiol M, Dabdoub A, Woods C, Kelley MW (2006) Inhibitors of differentiation and DNA binding (Ids) regulate Mathl and hair cell formation during the development of the organ of Corti. J Neurosci 26:550-558.

Kee Y, Bronner-Fraser M (2001a) Id4 expression and its relationship to other Id genes during avian embryonic development. Mech Dev 109: 341-345.

Kee Y, Bronner-Fraser M (2001b) Temporally and spatially restricted expression of the helix-loop-helix transcriptional regulator Id1 during avian embryogenesis. Mech Dev 109:331-335.

Kee Y, Bronner-Fraser M (2001c) The transcriptional regulator Id3 is ex- pressed in cranial sensory placodes during early avian embryonic development. Mech Dev 109:337-340.

Korchynskyi O, ten Dijke P (2002) Identification and functional characterization of distinct critically important bone morphogenetic proteinspecific response elements in the Id1 promoter. J Biol Chem 277:48834891.

León Y, Vazquez E, Sanz C, Vega JA, Mato JM, Giraldez F, Represa J, VarelaNieto I (1995) Insulin-like growth factor-I regulates cell proliferation in the developing inner ear, activating glycosyl-phosphatidylinositol hydrolysis and Fos expression. Endocrinology 136:3494-3503.

Li H, Corrales CE, Wang Z, Zhao Y, Wang Y, Liu H, Heller S (2005) BMP4 signaling is involved in the generation of inner ear sensory epithelia. BMC Dev Biol 5:16.

Massagué J, Seoane J, Wotton D (2005) Smad transcription factors. Genes Dev 19:2783-2810.

Matkowskyj KA, Schonfeld D, Benya RV (2000) Quantitative immunohistochemistry by measuring cumulative signal strength using commercially available software photoshop and matlab. J Histochem Cytochem 48: 303-312.

Morsli H, Choo D, Ryan A, Johnson R, Wu DK (1998) Development of the mouse inner ear and origin of its sensory organs. J Neurosci 18:33273335.

Neves J, Kamaid A, Alsina B, Giraldez F (2007) Differential expression of Sox 2 and Sox 3 in neuronal and sensory progenitors of the developing inner ear of the chick. J Comp Neurol 503:487-500.

Norton JD (2000) ID helix-loop-helix proteins in cell growth, differentiation and tumorigenesis. J Cell Sci 113:3897-3905.

Oh SH, Johnson R, Wu DK (1996) Differential expression of bone morphogenetic proteins in the developing vestibular and auditory sensory organs. J Neurosci 16:6463-6475.

Paizs M, Engelhardt JI, Siklós L (2009) Quantitative assessment of relative changes of immunohistochemical staining by light microscopy in specified anatomical regions. J Microsc 234:103-112.

Pujades C, Kamaid A, Alsina B, Giraldez F (2006) BMP-signaling regulates the generation of hair-cells. Dev Biol 292:55-67.

Ruzinova MB, Benezra R (2003) Id proteins in development, cell cycle and cancer. Trends Cell Biol 13:410-418.

Sahly I, El-Amraoui A, Abitbol M, Petit C, Dufier JL (1997) Expression of myosin VIIA during mouse embryogenesis. Anat Embryol (Berl) 196: $159-170$.

Sánchez-Calderón H, Francisco-Morcillo J, Martín-Partido G, HidalgoSánchez M (2007) Fgf19 expression patterns in the developing chick inner ear. Gene Expr Patterns 7:30-38.

Stone JS, Shang JL, Tomarev S (2003) Expression of Proxl defines regions of the avian otocyst that give rise to sensory or neural cells. J Comp Neurol 460:487-502.

Varga AC, Wrana JL (2005) The disparate role of BMP in stem cell biology. Oncogene 24:5713-5721.

Viñals F, Ventura F (2004) Myogenin protein stability is decreased by BMP-2 through a mechanism implicating Id1. J Biol Chem 279:45766- 45772.

Viñals F, Reiriz J, Ambrosio S, Bartrons R, Rosa JL, Ventura F (2004) BMP-2 decreases Mash1 stability by increasing Id1 expression. EMBO J 23:3527-3537.

Wilkinson DG, Nieto MA (1993) Detection of messenger RNA by in situ hybridization to tissue sections and whole mounts. Methods Enzymol 225:361-373.

Woods C, Montcouquiol M, Kelley MW (2004) Math1 regulates development of the sensory epithelium in the mammalian cochlea. Nat Neurosci 7:1310-1318.

Wu DK, Oh SH (1996) Sensory organ generation in the chick inner ear. J Neurosci 16:6454-6462.

Yokota Y (2001) Id and development. Oncogene 20:8290-8298.

Yu PB, Deng DY, Lai CS, Hong CC, Cuny GD, Bouxsein ML, Hong DW, McManus PM, Katagiri T, Sachidanandan C, Kamiya N, Fukuda T, Mishina Y, Peterson RT, Bloch KD (2008) BMP type I receptor inhibition reduces heterotopic [corrected] ossification. Nat Med 14:1363-1369.

Zhao H, Ayrault O, Zindy F, Kim JH, Roussel MF (2008) Post-transcriptional down-regulation of Atoh1/Math1 by bone morphogenic proteins suppresses medulloblastoma development. Genes Dev 22:722- 727.

Zheng JL, Gao WQ (2000) Overexpression of Math1 induces robust production of extra hair cells in postnatal rat inner ears. Nat Neurosci 3:580586. 